\title{
Isomalto-oligosaccharides from rice and their potential use as pharma-nutraceuticals in prevention of colon cancer
}

\author{
Vijitra Plongbunjong1, Santad Wichienchot ${ }^{1}$, Siribhorn Madla ${ }^{2}$, Pleumjit Bunyapipat ${ }^{3}$, \\ Knud Erik Bach Knudsen ${ }^{4}$ and Potchanapond Graidist ${ }^{2,5}$
}

${ }^{1}$ Interdisciplinary Graduate School of Nutraceutical and Functional Food (IGS-NFF), Prince of Songkla University, Hat Yai, Songkhla 90110, Thailand; ${ }^{2}$ Department of Biomedical Sciences, Faculty of Medicine, Prince of Songkla University, Hat Yai, Songkhla 90110, Thailand; ${ }^{3}$ Department of Pathology, Faculty of Medicine, Prince of Songkla University, Hat Yai, Songkhla 90110, Thailand; ${ }^{4}$ Department of Animal Science, Faculty of Science and Technology, Aarhus University, Tjele, Denmark; ${ }^{5}$ The Excellent Research Laboratory of Cancer Molecular Biology, Prince of Songkla University, Hat Yai, Songkhla 90110, Thailand

Corresponding Author: Graidist, Potchanapond, PhD, Department of Biomedical Sciences, Faculty of Medicine, Prince of Songkla University, Hat Yai, Songkhla 90110, Thailand

Submission Date: February $1^{\text {st }}$, 2019. Acceptance Date: June $27^{\text {th }}$, 2019. Publication Date: June $30^{\text {th }}, 2019$.

Citation: Plongbunjong V, Wichienchot S, Madla S, Bunyapipat P, Knudsen KEB, Graidist P: Isomalto-oligosaccharides from rice and their potential use as pharma-nutraceuticals in prevention of colon cancer. Functional Foods in Health and Disease 2019, 9(6):371-383. DOI: https://doi.org/10.31989/ffhd.v9i6.598

\begin{abstract}
Background: Isomalto-oligosaccharides (IMO) were enzymatically produced from rice (rIMO) alone or in combination with product (instant rice porridge mixed rIMO) demonstrated bifidogenic and butyrogenic effects in in vitro studies. The potential use of IMO from rice for pharma-nutraceuticals to prevent or risk reduction of colon cancer was further investigated in rats in this study.
\end{abstract}

Objective: To investigate potential use of IMO from rice on prevention or risk reduction of colon cancer and gut microbiota modulation in rats, colonic polyp formation, histological changes, gut microbiota modulation, and butyrogenic properties were evaluated.

Methods: An acute toxicity test was performed in ICR mice with a single dose of 2,000 $\mathrm{mg} / \mathrm{kg}$ of tested IMO. The effects of rIMO were performed in AOM/DSS-induced Wistar rats. The animals were divided in 8 groups and treated three times a week with vehicle, three doses of rIMO, cIMO, and product (instant rice porridge mixed rIMO) before and after carcinogen administration. Animals were sacrificed at the end of $19^{\text {th }}$ week.

Results: Oral administration at up to $2,000 \mathrm{mg} / \mathrm{kg}$ of rIMO and product containing rIMO could be considered safe. Additionally, the animals chemically induced with AOM/DSS and treated with rIMO $(1,500 \mathrm{mg} / \mathrm{kg})$ and product containing rIMO significantly lower the occurrence of colonic polyps about $60 \%$. There were no changes in other blood hematologic and biochemistry values but an improved gut barrier function when compared with animals in control and vehicle 
groups. AOM/DSS-induced rats supplemented with product containing rIMO could retard the reduction of beneficial bacteria and butyric acid production and thereby suppres the increase of harmful bacteria through the AOM/DSS post-induction phase.

Conclusion: The results of this in vivo study suggest that consumption of rIMO alone or in combination with brown rice porridge could potentially help protect histological changes and risk reduction of developing for colon cancer. IMO from rice has potential use for pharmanutraceuticals for risk reduction of colon cancer.

Keywords: colon cancer, isomalto-oligosaccharides, prebiotic, rice, gut modulation

\section{BACKGROUND}

The prebiotics most commonly used as food ingredients and generally recognized as safe (GRAS) are inulin, fructo-oligosaccharides (FOS), and galacto-oligosaccharides (GOS). However, other oligosaccharides, either natural origin (soy oligosaccharides (SOS), resistant starch, etc.) or enzyme/chemical-synthetic origin (isomalto-oligosaccharides (IMO), xylooligosaccharides (XOS), lactosucrose, pyrodextrin, polydextrose and lactulose) have been recently considered prebiotics and entered the world market [1]. IMOs are naturally found in various fermented foods and natural sweeteners. For commercial production of IMOs, starch derived from cereal crops and corn starch are primary source materials converted into a mixture of oligosaccharides with a degree of polymerization ranging from 2 to 10 using enzymatic technology [2, 3]. IMOs are known to be "bifidogenic" (promotion of the growth of bifidobacteria) in human volunteers [4-6] and are thereby regarded as potential prebiotics ingredients for the food, feed, and pharma-nutraceuticals industry. Nowadays, prebiotic sources are added as a supplement in various food products such as dairy products, beverages, health drinks, infant formulae, and meat products. The presence of prebiotics provides various health benefits such as improving calcium and magnesium absorption, increases bone density, reduces cancer risk, decreases cardiovascular diseases and also improves the immune system [7].

The properties on health-promoting benefits of IMO supplementation have been reported in both in vitro and in vivo studies. Some examples include the reduction of the risk of colon cancer, glycemic response and serum triglyceride reduction, intestinal epithelial ultrastructure conditions improvement, and beneficial gut microbe promotion [3]. Among these potential health claims, the use of IMO for developing ways to reduce the risk for colon cancer, are interesting because colon cancer is one of the global public health issues. The industrialization of the world and changes in lifestyle, including environmental and dietary factors, resulted in the steady increase the prevalent rates of colon cancer over the last century [8-10]. Colon cancer is considered as a preventable cancer. This is due to the epidemiological evidence that suggests that diets rich in plant-derived foods, including grains, fruits and vegetables, and that are low in red meat and fat, are associated with a reduced risk of developing colon cancer [11].

Rice is widely produced and a major exported agricultural product from Thailand. It is important for Thai people and many other Asian countries as their main source of food [12, 13]. Rice has been and continues to play a significant role in developing the Thai economy. Many rice-based products have been developed using science and technology, aiming to provide more income to Thai farmers. Two Thai rice-based products, rice-IMO (rIMO) and instant Sang-Yod brown rice porridge containing rIMO (ISYRP-mixed-rIMO), were developed in our previous study. Based on the results from in vitro studies, rIMO and ISYRP-mixed-rIMO demonstrated bifidogenic and butyrogenic effects, and also produced high levels of butyric acid [14, 15]. 
This study aimed to further investigate the potential health benefits of rIMO and rice porridge containing rIMO on preventing/reducing the risk of developing colon cancer in carcinogenesis rats.

\section{METHODS}

\section{Preparation of rIMO and product containing rIMO}

rIMO and product containing rIMO were prepared according to methods as previously described $[14,15]$. Commercial isomalto-oligosaccharides (cIMO) were purchased from Honne Co., Ltd. (Jiangsu, China).

\section{Animals and sample collection}

Male and female imprinting control region (ICR) mice of 8 weeks of age with a weight of 20-40 $\mathrm{g}$, were used for the acute toxicology study. Both male and female Wistar rats of 4 weeks of age with a weight of 140-180 g, were used in a study on the influence of rIMO and product containing rIMO in colon carcinogenesis rats.

The animals were obtained from and housed at the Southern Laboratory Animal Facility, Prince of Songkla University, Songkhla, Thailand. The animals were fed with food and water $a d$ libitum and acclimatized to laboratory conditions for 7 days prior to the experiment. The animals were maintained at a room temperature of $22 \pm 2{ }^{\circ} \mathrm{C}, 80 \pm 10 \%$ humidity, with a 12-hour light/dark cycle. All procedures performed in this study were approved by the Ethics Committee on Animal Experiment, Prince of Songkla University, Thailand (Ref 16/2015). The naturally passed feces were collected from each rat every week during the experimental period and kept at $-80^{\circ} \mathrm{C}$ until further analysis.

At the end of experiment, all animals were sacrificed, and the blood samples were collected for hematologic and biochemical analysis. The organs were weighed and calculated for the relative organ weight. The colon was removed and divided into 3 parts; proximal, middle and distal colon, presented at 0-20\%, 60-80 \% and 60-100\% of colon length, respectively [16]. The prevalence of colon polyps was detected on each part of colon and removed for hematoxylin and eosin (H\&E) staining.

\section{Acute toxicity study}

An acute toxicity test was performed according to the Organization of Economic Co-operation and Development (OECD) Guideline No.425 [17]. Forty ICR mice of both sexes were divided into five groups of eight animals each (four males and four females). Group 1 (control group) received normal saline (vehicle). Group 2 to 5 were orally treated with a single dose of 2,000 $\mathrm{mg} / \mathrm{kg}$ of cIMO, rIMO, rice porridge and rice porridge mixed rIMO, respectively. After administration, mice were observed for 24 hours, with special attention given to the first 4 hours and then daily for a period of 14 days for signs of toxicity. Mice were weighed and visualized observations of mortality, behavior, and changes in physical appearance, were conducted once daily during the experimental period.

\section{Effects of rIMO and product mixed rIMO consumption in colon carcinogenesis rats}

Forty-eight Wistar rats of both sexes were divided into eight groups, each group consisting of 6 animals (three males and three females). Group I (normal), rats were fed with food and water $a d$ libitum. Rats in Group II (negative control) received no treatment and Group III was treated with normal saline (vehicle). Group IV to VI, rats were orally administered with rIMO at 500, 1,000 and $1,500 \mathrm{mg} / \mathrm{kg}$, respectively. Rats in Group VII (cIMO) received 1,500 mg/kg of cIMO. Group VIII (product mixed rIMO), rats were orally treated with product mixed rIMO at $1,000 \mathrm{mg} / \mathrm{kg}$. 
At week 4 of the study, rats in Groups 2 to 8 were intraperitoneally injected with $20 \mathrm{mg} / \mathrm{kg}$ of azoxymethane (AOM). A week after AOM injection, rats were subjected to 4 cycles of dextran sodium sulphate (DSS) treatment. Each DSS-cycle consisted of replacing drinking water in cages with $3.5 \%$ DSS for 5 days, followed by a 7-day recovery period with regular water. Rats in Groups III to VIII were treated with vehicle, three doses of rIMO, cIMO and product mixed rIMO for three times in a week before and after AOM/DSS induction and remained the treatment procedure throughout 17 weeks of experimental period.

\section{Histopathologic study}

Each part of colon was fixed with $10 \%$ buffered formalin solution for 24 hours at room temperature. The specimens were rinsed in water and dehydrated in series of ethanol concentrations, followed by xylene and embedded in paraffin. Paraffin sections (3-5 $\mathrm{mm}$ ) were mounted on slides, deparaffinized and stained with hematoxylin and eosin. The histological evaluation was performed by professional pathologist.

\section{Short chain fatty acids (SCFA) and gut microbiota analysis}

The amount of SCFA and gut bacterial population contained in the feces, was determined by using HPLC and florescent in situ hybridization (FISH) technique with DNA probes targeted five groups of gut bacteria, respectively as previously described [14, 15]. Approximately $0.5 \mathrm{~g}$ of feces were diluted in distilled water, mixed and centrifuged at $5,000 \times \mathrm{g}$ for $5 \mathrm{~min}$. The supernatants were filtered with $0.45 \mu \mathrm{m}$ nylon filter paper prior to analysis for SCFA concentration.

\section{Statistical analysis}

All of the results were expressed as mean \pm standard error. The inter group variation was assessed by one-way analysis of variance (ANOVA) using SPSS software version 22 for Windows (New York, USA). For all significant results, post hoc comparison was performed using the statistical analysis. The Dunnett's $t$-test method and multiple comparisons were used for a detailed assessment of obtained data and assessment of their mutual relationship. The differences were considered significant $(p<0.05)$.

\section{RESULTS}

Acute toxicity test of rIMO and product mixed rIMO in mice

During the observation period, the behavioral patterns and physiological parameters (skin effects, hair loss, food intake, water consumption and excreta production) were normal with no signs of toxicity or mortality. Additionally, the hematologic and clinical blood chemistry values, body weights and internal organ weights were not significantly changed $(p>0.05)$ compared with the control group. Therefore, the results indicated that rIMO, cIMO, brown rice porridge, and brown rice porridge mixed rIMO could be considered safe for oral administration at up to $2,000 \mathrm{mg} / \mathrm{kg}$.

\section{Effects of rIMO and product mixed rIMO consumption in colon carcinogenesis rats}

\section{1) Food intake, body weight, hematologic and clinical blood chemistry parameters}

There was no significant difference $(p>0.05)$ in food intake, body weight and relative organ weight between the studied groups and control group (Table 1). For hematologic and clinical blood chemistry values, except blood platelet count, most of tested parameters were not statistically $(p>0.05)$ different (Table 2). 
Table 1. The mean body weight and the organ weight to body weight ratio from rats in different groups of treatment at sacrifice

\begin{tabular}{|c|c|c|c|c|c|c|c|c|}
\hline \multirow{2}{*}{ Group } & \multirow{2}{*}{$\begin{array}{l}\text { 24-h food intake } \\
\text { (wk17) (g/day) }\end{array}$} & \multirow{2}{*}{$\begin{array}{c}\text { Body weight } \\
\text { (g) }\end{array}$} & \multicolumn{6}{|c|}{ Organ weight/body weight (\%) } \\
\hline & & & Heart & Kidney & Liver & Lung & Spleen & Stomach \\
\hline Normal & $21.00 \pm 0.60$ & $389 \pm 13.04$ & $0.36 \pm 0.03$ & $0.71 \pm 0.12$ & $3.68 \pm 0.18$ & $0.51 \pm 0.03$ & $0.34 \pm 0.06$ & $1.46 \pm 0.23$ \\
\hline Control & $20.00 \pm 0.50$ & $353 \pm 12.45$ & $0.38 \pm 0.04$ & $0.69 \pm 0.09$ & $3.70 \pm 0.22$ & $0.51 \pm 0.10$ & $0.31 \pm 0.10$ & $1.59 \pm 0.28$ \\
\hline Vehicle & $20.60 \pm 0.60$ & $351 \pm 12.78$ & $0.37 \pm 0.02$ & $0.69 \pm 0.08$ & $3.66 \pm 0.15$ & $0.47 \pm 0.05$ & $0.29 \pm 0.05$ & $1.53 \pm 0.25$ \\
\hline rIMO-500 & $20.00 \pm 0.50$ & $380 \pm 12.49$ & $0.36 \pm 0.04$ & $0.70 \pm 0.13$ & $3.77 \pm 0.21$ & $0.52 \pm 0.08$ & $0.32 \pm 0.12$ & $1.61 \pm 0.31$ \\
\hline rIMO-1000 & $20.30 \pm 0.50$ & $395 \pm 12.31$ & $0.38 \pm 0.03$ & $0.68 \pm 0.09$ & $3.76 \pm 0.19$ & $0.50 \pm 0.07$ & $0.33 \pm 0.07$ & $1.53 \pm 0.24$ \\
\hline rIMO-1500 & $20.10 \pm 0.50$ & $387 \pm 14.22$ & $0.40 \pm 0.03$ & $0.65 \pm 0.04$ & $3.82 \pm 0.71$ & $0.49 \pm 0.05$ & $0.35 \pm 0.04$ & $1.49 \pm 0.26$ \\
\hline cIMO & $21.00 \pm 0.60$ & $390 \pm 13.61$ & $0.39 \pm 0.06$ & $0.66 \pm 0.07$ & $3.58 \pm 0.52$ & $0.52 \pm 0.08$ & $0.37 \pm 0.11$ & $1.60 \pm 0.29$ \\
\hline ISYRP-mixed-rIMO & $20.70 \pm 0.60$ & $385 \pm 12.33$ & $0.39 \pm 0.05$ & $0.70 \pm 0.06$ & $3.74 \pm 0.26$ & $0.55 \pm 0.12$ & $0.39 \pm 0.06$ & $1.64 \pm 0.30$ \\
\hline
\end{tabular}

Values are expressed as mean \pm SE. Means with the same letters are not significantly different $(p>0.05)$.

Table 2. The hematologic and clinical chemistry values from rats in different groups of treatment at sacrifice

\begin{tabular}{|c|c|c|c|c|c|c|c|c|}
\hline & \multirow{2}{*}{ Normal } & \multirow{2}{*}{ Control } & \multirow{2}{*}{ Vehicle } & \multicolumn{3}{|c|}{ rIMO } & \multirow{2}{*}{ cIMO } & \multirow{2}{*}{$\begin{array}{c}\text { ISYRP- } \\
\text { mixed-rIMO }\end{array}$} \\
\hline & & & & $500 \mathrm{mg} / \mathrm{kg}$ & $1,000 \mathrm{mg} / \mathrm{kg}$ & $1,500 \mathrm{mg} / \mathrm{kg}$ & & \\
\hline \multicolumn{9}{|l|}{ Hematologic values } \\
\hline White blood cells $\left(\times 10^{3} / \mu \mathrm{L}\right)$ & $2.48 \pm 0.16$ & $2.20 \pm 0.11$ & $2.23 \pm 0.19$ & $2.41 \pm 0.31$ & $3.03 \pm 0.27$ & $2.60 \pm 0.16$ & $2.39 \pm 0.40$ & $2.43 \pm 0.22$ \\
\hline Neutrophil (\%) & $47.13 \pm 5.13$ & $51.02 \pm 4.88$ & $49.10 \pm 6.12$ & $47.71 \pm 5.30$ & $50.32 \pm 3.35$ & $53.43 \pm 1.56$ & $47.35 \pm 3.55$ & $55.16 \pm 2.30$ \\
\hline Eosinophil (\%) & $0.00 \pm 0.00$ & $2.30 \pm 0.41$ & $2.00 \pm 0.30$ & $0.23 \pm 0.00$ & $0.00 \pm 0.00$ & $1.21 \pm 0.62$ & $0.00 \pm 0.00$ & $0.00 \pm 0.00$ \\
\hline Lymphocyte (\%) & $45.10 \pm 8.64$ & $41.36 \pm 5.05$ & $40.00 \pm 5.11$ & $42.76 \pm 7.30$ & $50.14 \pm 7.53$ & $47.21 \pm 3.37$ & $42.01 \pm 6.22$ & $50.02 \pm 8.01$ \\
\hline Monocyte (\%) & $0.00 \pm 0.00$ & $1.00 \pm 0.00$ & $1.05 \pm 0.21$ & $0.36 \pm 0.00$ & $1.04 \pm 0.00$ & $1.00 \pm 0.00$ & $0.00 \pm 0.00$ & $1.00 \pm 0.00$ \\
\hline Red blood cells $\left(\mathrm{x} 10^{6} / \mu \mathrm{L}\right)$ & $7.78 \pm 0.31$ & $7.42 \pm 0.67$ & $7.39 \pm 0.70$ & $7.77 \pm 0.06$ & $7.60 \pm 0.55$ & $7.80 \pm 0.35$ & $7.79 \pm 0.81$ & $7.91 \pm 0.44$ \\
\hline Hematocrit (\%) & $41.38 \pm 2.35$ & $40.07 \pm 3.48$ & $39.92 \pm 3.52$ & $43.39 \pm 3.10$ & $42.11 \pm 2.28$ & $41.90 \pm 2.87$ & $42.28 \pm 4.01$ & $43.01 \pm 2.48$ \\
\hline Hemoglobin $(\mathrm{g} / \mathrm{dL})$ & $13.41 \pm 0.43$ & $12.70 \pm 1.32$ & $12.72 \pm 1.00$ & $13.13 \pm 1.05$ & $13.14 \pm 0.53$ & $13.32 \pm 0.41$ & $13.13 \pm 1.14$ & $13.71 \pm 0.48$ \\
\hline $\mathrm{MCV}(\mathrm{fL})$ & $54.01 \pm 0.50$ & $53.71 \pm 1.10$ & $53.93 \pm 1.45$ & $54.21 \pm 1.50$ & $54.31 \pm 1.74$ & $53.94 \pm 0.77$ & $54.45 \pm 1.53$ & $53.80 \pm 0.71$ \\
\hline $\mathrm{MCH}(\mathrm{pg})$ & $17.72 \pm 1.00$ & $17.58 \pm 0.33$ & $17.60 \pm 0.56$ & $17.72 \pm 0.50$ & $17.53 \pm 1.00$ & $17.89 \pm 0.42$ & $17.80 \pm 0.31$ & $17.70 \pm 0.00$ \\
\hline $\mathrm{MCHC}(\mathrm{g} / \mathrm{dL})$ & $32.71 \pm 1.04$ & $31.46 \pm 1.00$ & $32.35 \pm 0.80$ & $32.11 \pm 1.00$ & $32.09 \pm 1.00$ & $32.31 \pm 0.36$ & $31.00 \pm 0.71$ & $32.21 \pm 0.26$ \\
\hline Platelet $\left(\times 10^{3} / \mu \mathrm{L}\right)$ & $661.15 \pm 35.60^{\mathrm{a}}$ & $596.33 \pm 13.79$ & $579.61 \pm 22.20$ & $546.63 \pm 19.30$ & $678.01 \pm 18.36^{\mathrm{a}}$ & $654.97 \pm 14.39^{\mathrm{a}}$ & $638.69 \pm 19.68^{\mathrm{a}}$ & $659.32 \pm 15.81^{\mathrm{a}}$ \\
\hline \multicolumn{9}{|l|}{ Clinical chemistry values } \\
\hline Cholesterol (mg/dL) & $45.71 \pm 2.14$ & $46.00 \pm 8.74$ & $45.10 \pm 5.29$ & $42.75 \pm 9.16$ & $49.04 \pm 4.42$ & $43.27 \pm 5.30$ & $47.33 \pm 9.18$ & $48.32 \pm 4.17$ \\
\hline Triglyceride (mg/dL) & $163.05 \pm 23.14$ & $159.31 \pm 17.11$ & $154.69 \pm 13.17$ & $157.65 \pm 21.35$ & $154.42 \pm 18.27$ & $151.33 \pm 20.85$ & $158.67 \pm 23.42$ & $157.10 \pm 22.35$ \\
\hline Total protein $(\mathrm{g} / \mathrm{dL})$ & $5.21 \pm 0.35$ & $5.37 \pm 0.53$ & $5.35 \pm 0.43$ & $5.37 \pm 0.31$ & $5.09 \pm 0.51$ & $5.28 \pm 0.20$ & $5.50 \pm 0.42$ & $5.60 \pm 0.72$ \\
\hline Albumin $(\mathrm{g} / \mathrm{dL})$ & $3.10 \pm 0.13$ & $3.11 \pm 0.20$ & $3.18 \pm 0.12$ & $3.23 \pm 0.12$ & $3.14 \pm 0.18$ & $3.13 \pm 0.11$ & $3.23 \pm 0.14$ & $3.17 \pm 0.22$ \\
\hline Aspartate aminotransferase (U/L) & $196.72 \pm 15.50$ & $194.62 \pm 15.94$ & $190.00 \pm 19.02$ & $195.33 \pm 13.23$ & $190.67 \pm 20.66$ & $203.33 \pm 13.09$ & $202.13 \pm 15.31$ & $207.00 \pm 23.16$ \\
\hline Alanine aminotransferase (U/L) & $68.33 \pm 6.93$ & $67.00 \pm 7.50$ & $68.00 \pm 11.12$ & $67.00 \pm 9.45$ & $67.67 \pm 1.57$ & $65.33 \pm 11.35$ & $65.00 \pm 6.20$ & $66.00 \pm 4.50$ \\
\hline Alkaline phosphatase (U/L) & $109.47 \pm 5.71$ & $102.02 \pm 12.31$ & $106.70 \pm 2.24$ & $113.21 \pm 9.19$ & $105.03 \pm 11.62$ & $109.05 \pm 11.31$ & $112.02 \pm 10.19$ & $105.13 \pm 12.00$ \\
\hline Total bilirubin (mg/dL) & $0.43 \pm 0.05$ & $0.37 \pm 0.09$ & $0.35 \pm 0.15$ & $0.47 \pm 0.37$ & $0.39 \pm 0.08$ & $0.45 \pm 0.05$ & $0.40 \pm 0.02$ & $0.41 \pm 0.15$ \\
\hline
\end{tabular}

Abbreviations: $\mathrm{MCV}$, mean corpuscular volume; $\mathrm{MCH}$, mean corpuscular hemoglobin; MCHC, mean corpuscular hemoglobin concentration.

Data are presented as means $\pm \mathrm{SEM},{ }^{\mathrm{a}} p<0.05$, compared to the control group.

\section{2) Histological results}

Colon tissue samples of rats in the normal group exhibited normal bowel structure with regular epithelial lines, intact crypts, and numerous goblet cells were detected in the surface and crypt 
epithelium (Figure $1 \mathrm{a}-\mathrm{c}$ ). The colon tissues of rats with rIMO (Figure $1 \mathrm{~g}$-i) and product mixed rIMO (Figure $1 \mathrm{j}-1$ ), intake had more visible goblet cells, intact crypts, and mucosal structure when compared to rats in control group (Figure $1 \mathrm{~d}-\mathrm{f}$ ). These results suggested that rIMO and product mixed rIMO could help reduce colon damages causing by AOM/DSS induction.
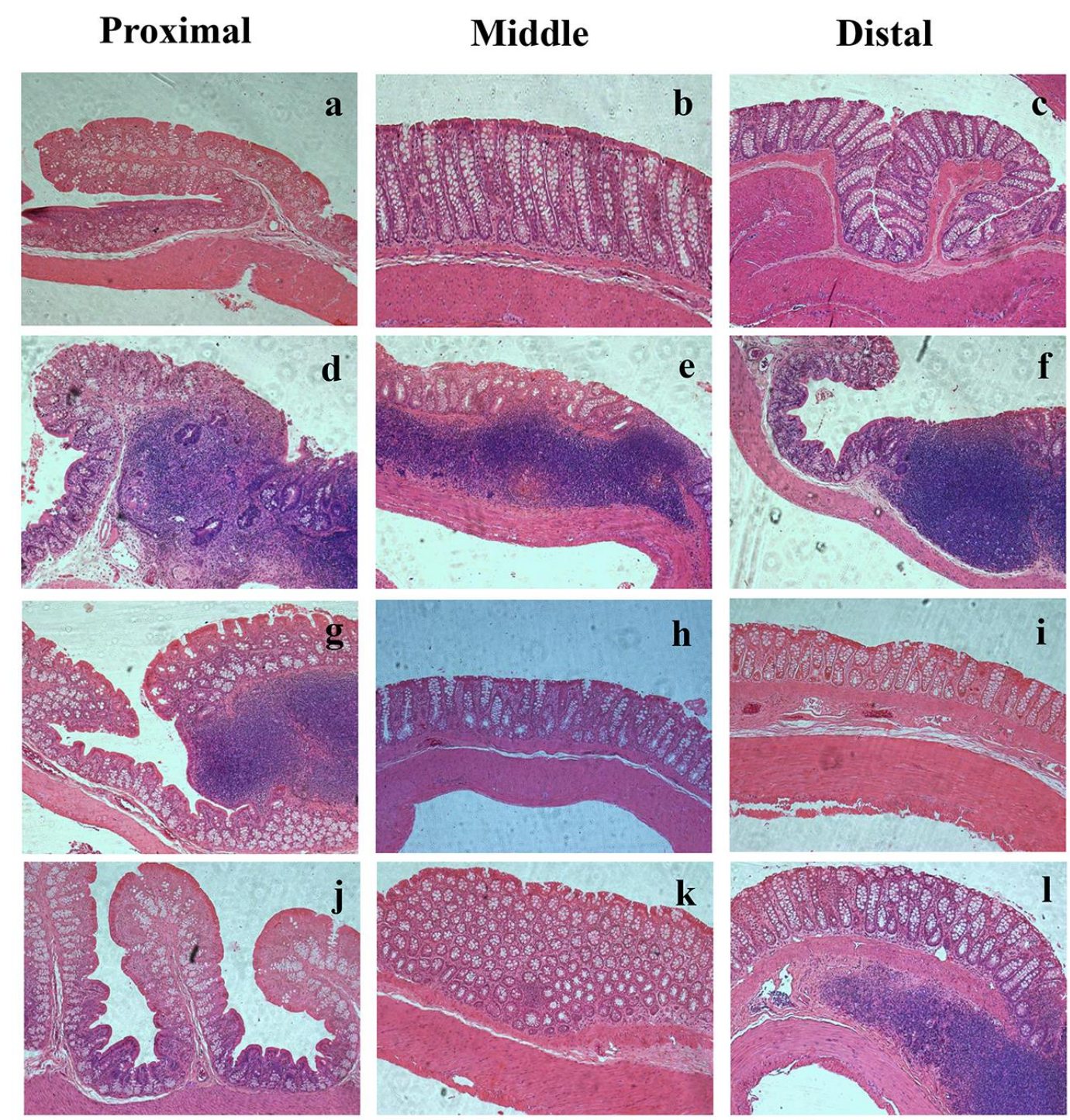

Normal
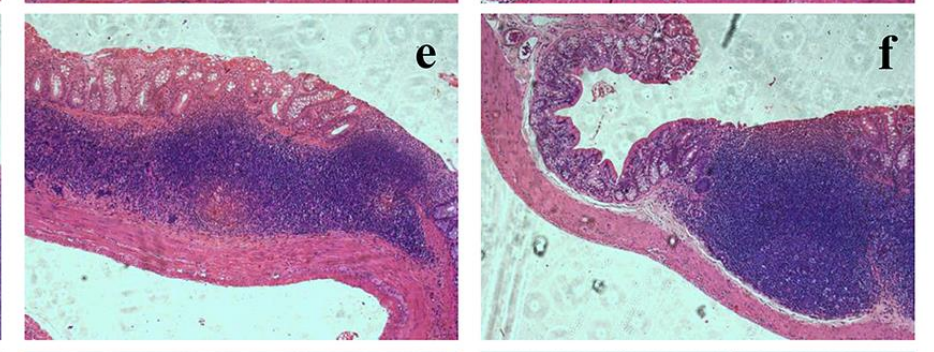

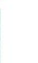

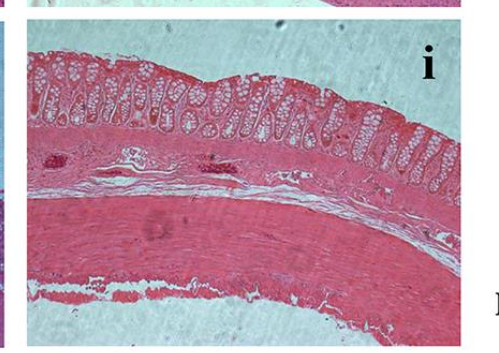

Control
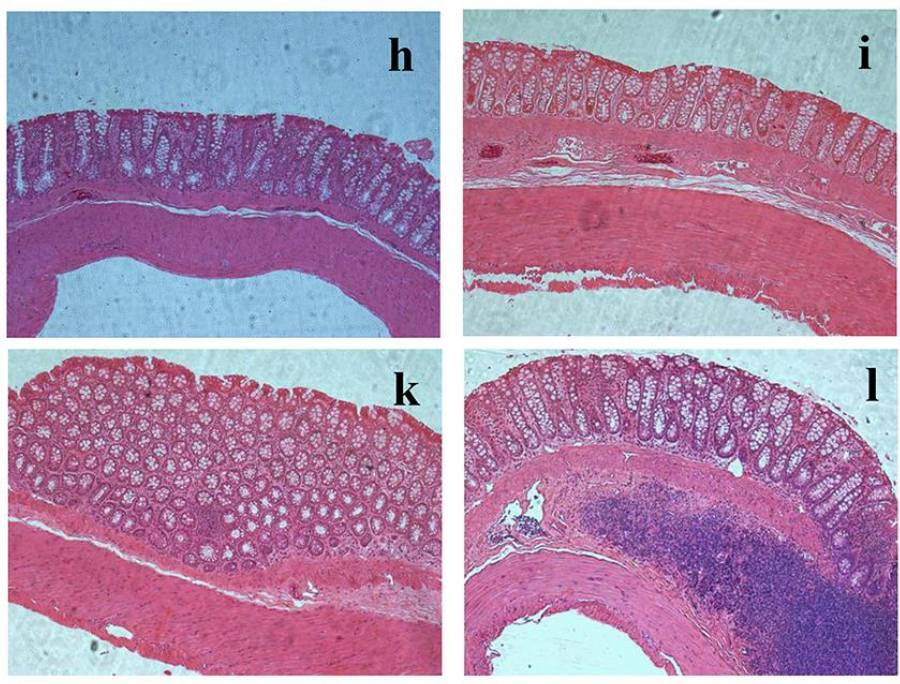

rIMO-1500

ISYRP-mixedrIMO

Figure 1. Histological analysis of colonic epithelium from rats in different treatment groups

\section{3) Polyps formation}

The prevalence of colonic polyps was considered an indicator for the initiation stage of the risk of colon cancer development. The effects of rIMO on the incidence and distribution of polyps in different part of colons were presented in Table 3. There was no polyp developed in normal rats. The highest number of polyps (23.83) was observed in the control group with the distribution of polyps formed in proximal $(9.17 \pm 0.75)$, middle $(5.33 \pm 1.21)$, and distal $(9.33 \pm 1.63)$. The administration of rIMO significantly reduced $(p<0.05)$ the numbers of polyp developed in rats compared to the control group. The minimum percentage of polyp incidence was detected in rats treated with rIMO-1500 (38.48\%), followed by cIMO-1500 (39.15\%) and product mixed rIMO $(41.96 \%)$. 
Table 3. The occurrence of colonic polyps from rats in different groups of treatment at sacrifice

\begin{tabular}{lrrrrr}
\hline \multirow{2}{*}{ Group } & \multicolumn{3}{c}{ Numbers of colonic polyps } & \multicolumn{2}{c}{$\begin{array}{c}\text { Occurrence of colonic } \\
\text { polyps (\%) }\end{array}$} \\
\cline { 2 - 5 } Normal & Proximal & \multicolumn{1}{c}{ Middle } & \multicolumn{1}{c}{ Distal } & Total & 0 \\
Control & 0 & 0 & 0 & 0 & 100 \\
Vehicle & $9.17 \pm 0.75$ & $5.33 \pm 1.21$ & $9.33 \pm 1.63$ & $23.83 \pm 2.79$ & 93.03 \\
rIMO-500 & $9.00 \pm 1.41$ & $5.50 \pm 1.05$ & $7.67 \pm 1.03$ & $22.17 \pm 0.75$ & 84.64 \\
rIMO-1000 & $8.67 \pm 1.21$ & $5.33 \pm 0.82$ & $6.17 \pm 1.17$ & $20.17 \pm 1.83$ & 71.34 \\
rIMO-1500 & $6.33 \pm 1.21$ & $5.17 \pm 0.98$ & $5.50 \pm 0.55$ & $17.00 \pm 2.19$ & 38.48 \\
cIMO & $6.17 \pm 1.07$ & $1.33 \pm 0.47$ & $1.67 \pm 0.75$ & $9.17 \pm 1.17$ & 39.15 \\
ISYRP-mixed-rIMO & $4.17 \pm 0.90$ & $1.33 \pm 0.47$ & $4.50 \pm 0.50$ & $10.00 \pm 1.41$ & 41.96 \\
\hline
\end{tabular}

\section{4) Gut microbiota population and SCFA production}

Before carcinogen (AOM/DSS) induction, the continuous administration of rIMO-1500 could predominantly increase numbers of Lactobacillus and Bifidobacterium in rat fecal samples compared to other experimental groups (Figure 2).
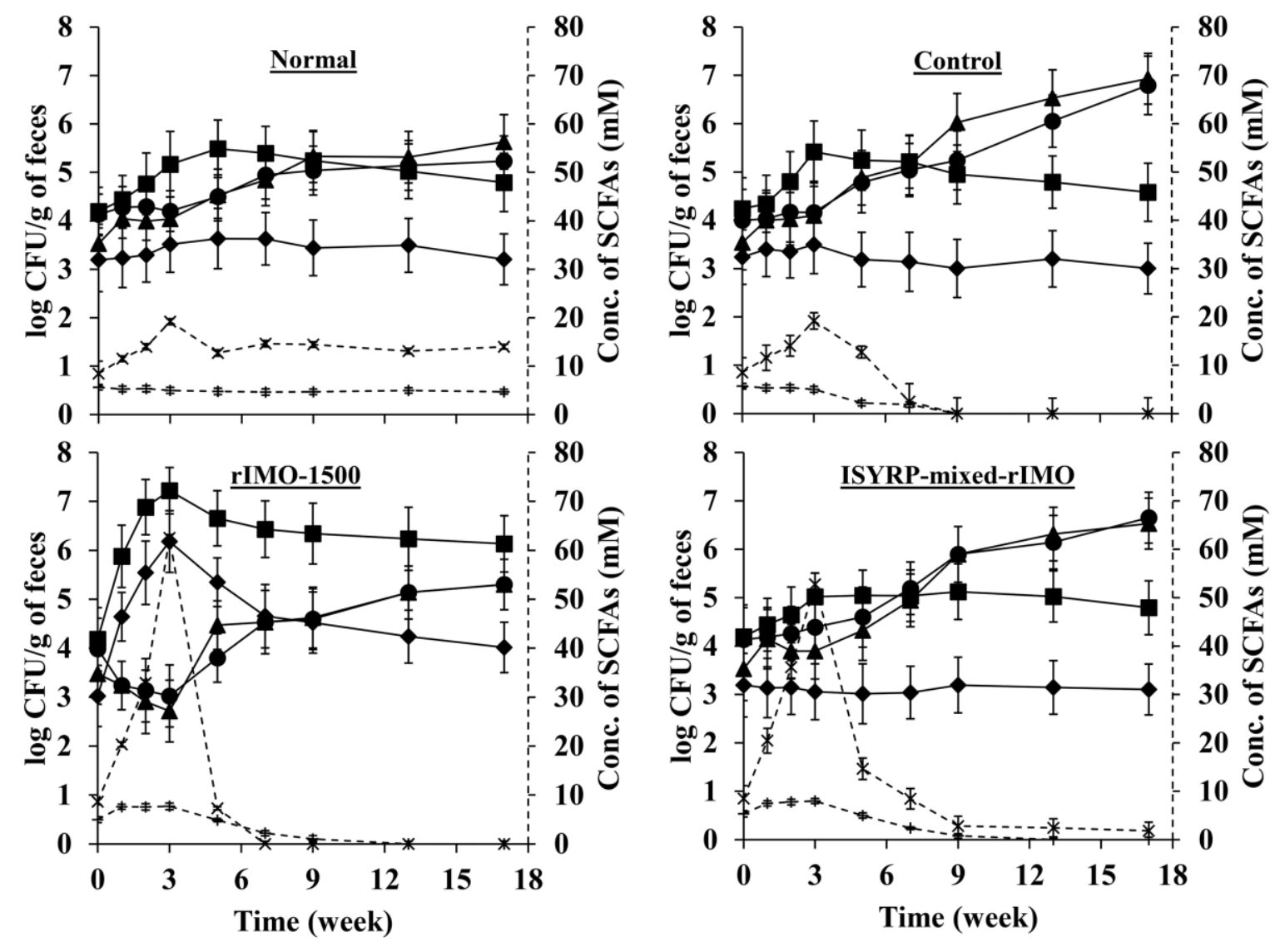

Figure 2. The concentration of bifidobacteria $\left(--_{-}\right)$, lactobacilli $\left(-\boldsymbol{\square}_{-}\right)$, clostridia $\left(-\boldsymbol{\Delta}_{-}\right)$, bacteroides (-- - ), acetic acid (---+--) and butyric acid (---X--) containing in feces from rats in different treatment groups during the experimental period 
Lactobacillus and Bifidobacterium counts reached their maximum on the $3^{\text {rd }}$ week and then declined continuously after AOM/DSS induction. Moreover, all studied groups had greater numbers of Lactobacillus than Bifidobacterium counts. During the observation period, only small changes in the number of Bifidobacterium were observed in rat fecal samples from the normal, control, and product mixed rIMO groups.

In this study, only acetic acid and butyric acid were detected in rat fecal samples (Figure 2). The concentrations of butyric acid significantly increased $(p<0.05)$ in rats receiving rIMO-1500 and product mixed rIMO and reached the highest amount (62.54 and $52.76 \mathrm{mM}$ respectively) on the $3^{\text {rd }}$ week and decreased continuously after AOM/DSS induction.

\section{DISCUSSION}

In vitro fecal fermentation of rIMO and product mixed rIMO studies demonstrated that rIMO could be an alternative source of prebiotic dietary fiber as it promoted the growth of bifidobacteria and increased butyric acid production. Those findings have gained significant attention as they play an important role in colon cancer prevention [14, 15]. Therefore, the present study was conducted to evaluate health benefits of rIMO and brown rice porridge mixed rIMO in the colitis-associated colon carcinogenesis rats using AOM/DSS-induced model. AOM/DSS is a two-stage colon tumor model to mimic colitis-driven tumor development that can induce colon tumor in a short-term period and has been used for chemoprevention studies of colitis-associated colon cancer [18, 19].

A number of experimental studies indicated the beneficial effects of prebiotic oligosaccharides supplementation such as FOS, XOS, and GOS, in a rodent colon carcinogenesis model using different rodent strains, protocol of tumor induction and biomarkers for colon cancer [20-23]. Aberrant crypt foci (ACF) are considered and have been widely used as cancer endpoints to evaluate anti-cancer compounds because of their chemoprevention potential for colon cancer at an early stage. The best endpoints as tumors are long-term carcinogenesis experiments. However, these experiments require more time and animal subjects compared to ACF [21]. In this study, the incidence of colonic polyps was used to assess the protective effects of rIMO and product mixed rIMO against colon cancer development in AOM/DSS-treated rats.

Genetic susceptibility of rodent strains and protocol of tumor induction play an important role in the risk for colorectal tumorigenesis, resulting in different tumor development stages ranging from dysplastic epithelium, progressive adenoma to carcinoma in situ and malignant growth [24]. Dysplasia has the potential to develop into a polyp, which is a nonmalignant adenoma that grows from hyperproliferative epithelium region and protrudes into the colonic lumen [25]. The presence of colonic polyp is one of the factors that may affect the risk of colon cancer development, concerning regard to the transition of polyps to cancer $(<1 \%)$ over time [26]. Based on our data, no histological changes were observed in normal rats through the observation period. Some histological changes, such as polyp formation, infiltration of 
inflammatory cells, destruction of crypts, and goblet cells depletion were detected in rats treated with AOM/DSS. The supplementation with rIMO in AOM/DSS-treated rats, tended to result in the inhibition of the incidence of polyps in dose-dependent manner, while those with no supplementation with rIMO (control and vehicle groups) tended to have more polyp formation in the colon. The administration of rIMO alone and in combination with brown rice porridge could help the reduction of intestinal damages and decrease of the polyp incidences (about $60 \%$ were found in control and vehicle groups). Some of the polyps were further examined by $H \& E$ staining and were classified as tubular adenoma, pre-cancerous with 5\% carrying a risk of becoming cancerous [27]. Moreover, treatment of rIMO and product mixed rIMO helped improve conditions of the intestinal epithelium caused by AOM/DSS administration compared to the experimental group without rIMO treatment. The reduction of colon damages effects agreed with those reported for the effects of inulin-type fructan in colon cancer rats by Rivera-Huerta and colleagues [23]. Thus, we can speculate that there was a positive relationship between consumption of rIMO and brown rice porridge mixed rIMO and its contribution in the risk reduction of colon cancer.

Another health benefit of the consumption of prebiotics is modulating gut microbiota and promoting beneficial bacteria, Bifidobacterium and Lactobacillus, which lead to the increase of the production of SCFA particular butyric acid. For example, consumption of prebiotic oligosaccharides, galacto-oligosaccharides (GalOS, 76-151 mg) in dihydromethysticin-induced Wistar rats for 16 weeks demonstrated the reduction of ACF and harmful bacteria, while the populations of beneficial bacteria and SCFA production increased [20]. In this study, the bifidogenic and butyrogenic effects of rIMO in AOM/DSS-induced rats were consistent with the previous reports. According to the study about the changes in microbial community in AOM/DSS-induced C57BL/6 male mice by Zackular and colleagues [28], they reported that both AOM-induced DNA damage and DSS-induced inflammatory caused microbial community changes, while each round of DSS treatment resulted in a significant change in the structure of the microbiome. Specifically, AOM/DSS caused the reduction in operational taxonomic units within the family of Porphyromonadaceae, the important mediators of anti-inflammatory signals in the gut. The study from Zackular and colleagues supported our results, as consumption of rIMO displayed bifidogenic and butyrogenic effects in rats during the pre-induction of AOM/DSS.

During the post-induction of AOM/DSS, rats continually daily treated with rIMO-1500 showed small difference in the growth of Bifidobacterium ( 10 times higher) and Lactobacillus ( 100 times higher) compared to rats in the control group. During the trial, butyric acid was dramatically decreased after AOM injection and finally disappeared from the feces of rats in control and rIMO-1500 groups on the $9^{\text {th }}$ week of observation period. AOM/DSS-induced rats supplemented with product mixed rIMO demonstrated a higher number of beneficial bacteria than harmful bacteria (Figure 3 and butyric acid still presented in a small amount through the 
AOM/DSS post-induction phase. The effective dose for rIMO and product mixed rIMO might higher than fructan-type prebiotics [14].

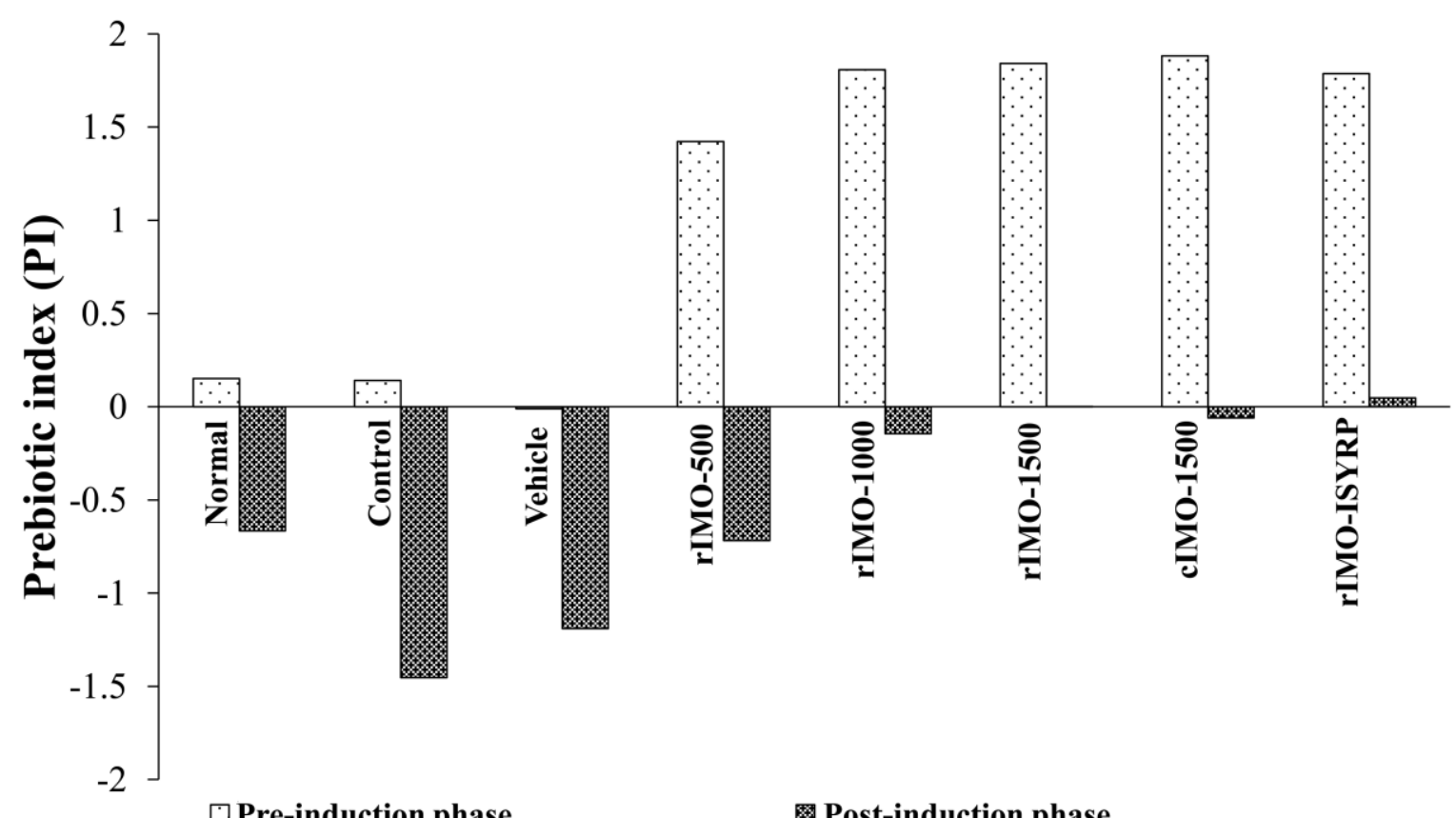

Figure 3. Prebiotic index (PI) from different treatment groups in the AOM/DSS pre-induction (week 3) and AOM/DSS post-induction (week 17) phases. The PI was calculated according to the equation; PI $=[($ Bif $/$ total $)+($ Lac/total $)]-[($ Bac/total $)+($ Clos/total $)]$. Where Bif is bifidobacterial numbers at sample time/number at inoculation; Lac is lactobacilli numbers at sample time/number at inoculation; Bac is bacteroides numbers at sample time/number at inoculation; Clos is clostridia numbers at sample time/number at inoculation; total is total bacterial numbers at sample time/number at inoculation

\section{CONCLUSIONS}

Consumption of rIMO alone or in combination with brown rice porridge can inhibit or prevent the initiation of cells in the colon into pre-neoplastic cells, which were caused by AOM/DSS induction. From the results of the study we concluded that rIMO consumption reduced several histological changes (polyp formation and epithelial structure) in the colon of AOM/DSS-treated rats. The possible roles for rIMO could be to improve colonic peristalsis induced by SCFA produced, thereby helping food move through colon faster and reducing the amount and intact time of carcinogens in the colon. Additionally, rIMO could regulate gut dysbiosis and strengthen tight-junction by promoting the growth of Akkermansia muciniphila, resulting in the prevention of gut-leak, reducing mucosal inflammation, immune modulation, and reduce risk of colon cancer. This means that IMO from rice has potential use as pharma-nutraceuticals for prevention or risk reduction of colon cancer.

However, in order to understand the clear mechanism of rIMO on initiation of colon cancer prevention, the inflammation-dysplasia-carcinoma carcinogenesis process such as the levels of proinflammatory cytokines/chemokines, and the infiltration of inflammatory cell (macrophages, neutrophils) need to be further investigated. 
Competing Interests: The authors declare that there is no conflict of interest.

Author's Contributions: All authors contributed to these studies.

Acknowledgements and Funding: This work was financially supported from The Royal Golden Jubilee Ph.D. Programme Scholarships (PHD/0005/2556), Thailand, PSU Research Fund [MED 601340S] and the Excellence Research Laboratory (ExLab004), Prince of Songkla University, Hat Yai, Songkhla, Thailand.

\section{REFERENCES}

1. Younis K, Ahmad S, Jahan K: Health benefits and application of prebiotics in foods. J Food Process Technol 2015, 6:1-7.

2. Isomalto-oligosaccharide (VitaFiber): Novel Food Information-Isomaltooligosaccharide (VitaFiber ${ }^{\mathrm{TM}}$ ). Retrieved from [https://www.canada.ca/en/healthcanada/services/food-nutrition/genetically-modified-foods-other-novel-foods/approvedproducts/isomalto-oligosaccharide-vitafiber-trade.html] Retrieved October 11, 2012

3. Sorndech W, Na Nakorn K, Tongta S, Blennow A: Isomalto-oligosaccharides: recent insights in production technology and their use for food and medical applications. LWT - Food Sci Technol 2018, 95:135-142.

4. De Boever P, Deplancke B, Verstraete W: Fermentation by gut microbiota cultured in a simulator of the human intestinal microbiol ecosystem is improved by supplementing a soygerm powder. J Nutr 2000, 130(10):2599-2606.

5. Hayakawa K, Mizutani J, Wada K, Masai T, Yoshihara I, Mitsuoka T: Effect of soybean oligosaccharides on human faecal flora. Microb Ecol Health Dis 1990, 3(6):293-303.

6.Kohmoto T, Fukui F, Takaku H, Mitsuoka T: Dose-response test of isomaltooligosaccharides for increasing fecal bifidobacteria. Agric Biol Chem 1991, 55(8):2157-2159.

7. Ashwini A, Ramya HN, Ramkumar C, Reddy KR, Kulkarni RV, Abinaya V, Naveen S, et al.: Reactive mechanism and the applications of bioactive prebiotics for human health: Review. J Microbiol Meth 2019, 159:128-137.

8. Arnold M, Sierra MS, Laversanne M, Soerjomataram I, Jemal A, Bray F: Global patterns and trends in colorectal cancer incidence and mortality. Gut 2017, 66(4):683691.

9. Favoriti P, Carbone G, Greco M, Pirozzi F, Pirozzi RE, Corcione F: Worldwide burden of colorectal cancer: a review. Updates Surg 2016, 68(1):7-11.

10. Goncalves P, Martel F: Regulation of colonic epithelial butyrate transport: focus on colorectal cancer. Porto Biomed J 2016, 1(3):83-91.

11. Mutanen M, Pajari AM, Oikarinen SI: Beef induces and rye bran prevents the formation of intestinal polyps in $\mathrm{Apc}(\mathrm{Min})$ mice: relation to $\beta$-catenin and $\mathrm{PKC}$ isozymes. Carcinogenesis 2000, 21(6):1167-1173. 
12. Ricepedia. The global staple. Retrieved from http://ricepedia.org/rice-as-food/theglobal-staple-rice-consumers.

13. Thailand's Top 10 Exports-World's Top Exports [http://www.worldstopexports.com/ thailands-top-10-exports/] Retrieved September 1, 2019

14. Plongbunjong V, Graidist P, Bach Knudsen KE, Wichienchot S: Isomaltooligosaccharide synthesised from rice starch and its prebiotic properties in vitro. Int J Food Sci Technol 2017, 52(12):2589-2595.

15. Plongbunjong V, Graidist P, Bach Knudsen KE, Wichienchot S: Starch-based carbohydrates display the bifidogenic and butyrogenic properties in $\mathrm{pH}$-controlled feacal fermentation. Int J Food Sci Technol 2017, 52(12):2647-2653.

16. Talbot C, Lytle $\mathrm{C}$ : Segregation of $\mathrm{Na} / \mathrm{H}$ exchanger-3 and $\mathrm{Cl} / \mathrm{HCO} 3$ exchanger SLC26A3 (DRA) in rodent cecum and colon. Am J Physiol Gastrointest Liver Physiol 2010, 299(2):G358-367.

17. OECD: Test No. 425: Acute Oral Toxicity: Up-and-Down Procedure. OECD Guidelines for the Testing of Chemicals, Section 4. Paris: OECD; 2008.

18. Tanaka T: Development of an inflammation-associated colorectal cancer model and its application for research on carcinogenesis and chemoprevention. Int J Inflam 2012, 658786:1-16.

19. Ishikawa T, Herschman HR: Tumor formation in a mouse model of colitis-associated colon cancer does not require COX-1 or COX-2 expression. Carcinogenesis 2010, 31(4):729-736.

20. Qamar TR, Iqbal S, Syed F, Nasir M, Rehman H, Iqbal MA, Liu RH: Impact of novel prebiotic galacto-oligosaccharides on various biomarkers of colorectal cancer in Wistar rats. Int J Mol Sci 2017, 18(9):1-12.

21. Femia AP, Salvadori M, Broekaert WF, Francois IE, Delcour JA, Courtin CM, Caderni G: Arabinoxylan-oligosaccharides (AXOS) reduce preneoplastic lesions in the colon of rats treated with 1,2-dimethylhydrazine (DMH). Eur J Nutr 2010, 49(2):127-132.

22. Hijova E, Szabadosova V, Shtofilova J, Hrchkova G: Chemopreventive and metabolic effects of inulin on colon cancer development. J Vet Sci 2013, 14(4):387-393.

23. Rivera-Huerta M, Lizarraga-Grimes VL, Castro-Torres IG, Tinoco-Mendez M, MaciasRosales L, Sanchez-Bartez F, Tapia-Perez GG, et al.: Functional effects of prebiotic fructans in colon cancer and calcium metabolism in animal models. Biomed Res Int 2017, 2017:1-10.

24. De Robertis M, Massi E, Poeta ML, Carotti S, Morini S, Cecchetelli L, Signori E, et al.: The AOM/DSS murine model for the study of colon carcinogenesis: from pathways to diagnosis and therapy studies. J Carcinog 2011, 10:1-22.

25. Brennan CA, Garrett WS: Gut microbiota, inflammation, and colorectal cancer. Annu Rev Microbiol 2016, 70:395-411. 
26. Sivieri K, Bedani R, Cavallini DCU, Rossi EA: Prebiotics and intestinal microbiota: implications in colon cancer prevention. In Lactic Acid Bacteria - R and D for Food, Health and Livestock Purposes. Edited by Kongo. IntechOpen; 2013:217-242.

27. Bujanda L, Cosme A, Gil I, Arenas-Mirave JI: Malignant colorectal polyps. World J Gastroenterol 2010, 16(25):3103-3111.

28. Zackular JP, Baxter NT, Iverson KD, Sadler WD, Petrosino JF, Chen GY, Schloss PD: The gut microbiome modulates colon tumorigenesis. MBio 2013, 4(6):1-9. 